\title{
Changes in histone acetylation as potential mediators of pupal diapause in the flesh fly, Sarcophaga bullata
}

J. A. Reynolds ${ }^{1, *}$ Robin Bautista-Jimenez ${ }^{1}$, and D.L. Denlinger ${ }^{1,2}$

${ }^{1}$ Department of Evolution, Ecology, and Organismal Biology, The Ohio State University, Columbus $\mathrm{OH} 43210$, USA

${ }^{2}$ Department of Entomology, The Ohio State University, Columbus OH 43210, USA

Correspondence to:

Julie A Reynolds

Department of Evolution, Ecology, and Organismal Biology

318 W $12^{\text {th }}$ Ave.

300 Aronoff Laboratory

Columbus $\mathrm{OH} 43210$ USA

Phone: (614)-292-7287

Email: Reynolds.473@osu.edu 


\section{Abstract}

The growing appreciation that epigenetic processes are integral to the responses of many organisms to changes in the environment suggests a possible role for epigenetics in coordination of insect diapause. The results we present suggest that histone modification may be one type of epigenetic process that contributes to regulation of pupal diapause in the flesh fly, Sarcophaga bullata. Reduction in total histone H3 acetylation in diapausing pupae, shifts in mRNA expression profiles of genes encoding histone acetyltransferase (HAT) and histone deacetylase (HDAC) in pre-diapause, diapause and post-diapause flies compared to their nondiapause counterparts, and alterations in HDAC enzyme activity during and post-diapause lend support to the hypothesis that this specific type of histone modification is involved in regulating diapause programming, maintenance, and termination. Transcription of genes encoding HDAC1, HDAC3, HDAC6, and Sirtuin2 were all upregulated in photosensitive first instar larvae programmed to enter pupal diapause, suggesting that histone deacetylation may be linked to the early decision to enter diapause. A 50\% reduction in transcription of hdac 3 and a corresponding $30 \%$ reduction in HDAC activity during diapause suggest that removal of acetyl groups from histones primarily occurs prior to diapause entry and that further histone deacetylation is not necessary to maintain diapause. Transcription of the HDAC genes was quickly elevated when diapause was terminated, followed by an increase in enzyme activity after a short delay. A maternal effect operating in these flies prevents pupal diapause in progeny whose mothers experienced pupal diapause, even if the progeny are reared in strong diapause-inducing short-day conditions. Such nondiapausing pupae had HDAC transcription profiles nearly identical to the profiles seen in nondiapausing pupae generated under a longday photoperiod. Together, these results provide consistent evidence for histone acetylation and deacetylation as regulators of this insect's developmental trajectory.

Key words: Epigenetics, maternal effect, histone modification, phenotypic plasticity 


\section{Introduction}

Epigenetic mechanisms are increasingly recognized as factors used by organisms to sense and respond to changing environmental conditions (Kilvitis et al., 2014, Furusawa and Kaneko, 2013; Jaenisch and Bird, 2003). Broadly defined, epigenetic mechanisms are those that influence gene expression and alter phenotype independently of changes in DNA sequence. The best known epigenetic processes include DNA methylation, small RNA-interference, and histone post-translational modifications (PTMs). Independently or together, these processes generate a feed-back loop that provides short-term adjustments and long-term adaptations to environmental insult.

Histone acetylation is a specific type of histone PTM (i.e., one type of epigenetic mechanism) that is involved in numerous biological functions including circadian rhythms (Fogg et al., 2014; Malapeira et al., 2012), metabolism (Cosentino and Mostoslavsky, 2013; Kamemura et al., 2012; Katada et al., 2012; Karpac and Jasper, 2011; Chen et al., 2008), cellcycle regulation (Yamaguchi et al., 2010), and stress-response (Svetec et al., 2009; Matthias et al., 2008). This dynamic PTM mediates these diverse functions through coordinated, yet opposing, actions of histone acetyltransferases (HATs) and histone deacetylases (HDACs) which transcriptionally alter gene expression patterns by modifying chromatin structure to make specific sequences more (HATs) or less (HDACs) accessible to gene transcription machinery. Although numerous reports describe biological functions of histone acetylation/deacetylation, it is not clear how this process contributes to a change in phenotype or how it might promote adaptation to changing environmental conditions.

Our goal was to examine a possible role for histone acetylation/deacetylation in the regulation of insect diapause, an alternative developmental pathway that provides insects and other animals a mechanism for surviving seasonal periods of extreme environmental conditions. Insects entering diapause sense a token cue (e.g. change in photoperiod or decline in food quality) that signals the coming of unfavorable conditions and initiates a developmental pathway that includes a period of dormancy. The dormant period is characterized by developmental arrest, metabolic downregulation, and increased stress-resistance. These diapause-related changes are associated with wide-spread changes in gene expression patterns 
(Denlinger, 2002) that have been characterized for a variety of insects and other arthropods including spider mites (Byron et al., 2013), crickets (Reynolds and Hand, 2009), moths (Wadsworth and Dopman, 2015), solitary bees (Yocum et al., 2015 ), bumble bees (Amsalem et al. 2015), mosquitoes (e.g. Poelchau et al., 2013; Robich et al., 2007, Tormey et al., 2015), and several of the higher Diptera (Ragland et al., 2010, Ragland et al., 2011, Flannagan et al. 1998, Kubrak et al. 2014). Although we now have a fairly comprehensive overview of the types of changes in gene expression that contribute to the diapause phenotype, the processes that regulate diapause-related shifts in expression remain poorly understood. We predict that epigenetic mechanisms mediate changes in gene expression that contribute to the diapause phenotype. Our previous research provides evidence that small regulatory RNAs, another type of epigenetic process, contribute to pupal diapause regulation in the flesh fly, Sarcophaga bullata (Reynolds et al., 2013), and it is likely that other epigenetic processes are also involved. The present research focuses on the contribution of histone acetylation, one type of histone PTM, in generating the diapause phenotype. Histone deacetylation appears to mediate transcriptional repression in hibernating ground squirrels (Morin and Storey, 2006) and in aestivating desert frogs (Hudson et al, 2008). In addition, histone methylation, another type of PTM, serves as a regulator of pupal diapause in Helicoverpa armigera (Lu et al., 2013), and in embryonic diapause in annual killifish (Toni and Padilla, 2016). Though these four studies provide some evidence that histone modification mediates dormancy, the roles of histone acetylation in initiating, maintaining, and terminating insect diapause remain unknown.

This study investigates the contribution of histone acetylation in establishing, maintaining, and terminating pupal diapause in the flesh fly, Sarcophaga bullata, an established model for diapause research (e.g. Denlinger et al, 1972; Michaud and Denlinger, 2007; Rinehart et al, 2007; Hahn et al., 2009; Ragland et al., 2010; Rinehart et al., 2010). We predict that histone acetylation, an indicator of gene activity, will be reduced during diapause because gene silencing (i.e. repression of transcription) is a common feature of this dormant stage. We anticipate that HDAC enzyme activity and transcription of HAT and HDAC-encoding genes will correlate with the predicted reduction in histone acetylation. 
In addition, we investigated the possible contribution of HDACs to expression of a maternal effect that regulates entry into diapause in S. bullata. In this species, individuals that are the offspring of females that experienced pupal diapause are unable to enter diapause themselves (Henrich and Denlinger, 1982; Rockey et al., 1989). Assessing differences in the transcription of HDAC genes in photosensitive larvae and pupae from mothers with no diapause history compared to those from mothers that experienced diapause (i.e. have a diapause history) can potentially offer new regulatory insights on the regulation of diapause and this intriguing maternal effect.

\section{Methods}

\subsection{Insect Rearing}

S. bullata, from our established colony collected originally in Columbus, Ohio, were reared as described (Denlinger, 1972; Reynolds et al., 2013). Adults were kept at $25^{\circ} \mathrm{C}$ under either a short-day photoperiod ( $9 \mathrm{~h}$ photophase) to induce diapause in their progeny or under a long-day photoperiod (15 h photophase) to prevent diapause induction. Larvae were reared at $18^{\circ} \mathrm{C}$ under the same short-day or long-day photoperiod regime as their parents to promote (short-day) or prevent (long-day) diapause entry.

To assess changes associated with diapause development we sampled diapausing pupae at two time points. Early diapause (ED) flies were maintained at $18{ }^{\circ} \mathrm{C}$ for approximately $15 \mathrm{~d}$ post-pupariation; late diapause (LD flies) were sampled approximately $30 \mathrm{~d}$ post-pupariation. Nondiapause pupae were sampled when they reached the phanerocephalic stage (approximately $6 \mathrm{~d}$ post-larviposition at $18^{\circ} \mathrm{C}$ ), which is the developmental stage when $S$. bullata enters diapause.

Post-diapause flies were generated by removing the anterior cap of the puparium and applying $5 \mu$ l hexane to the exposed head, a treatment that terminates diapause (Denlinger et al., 1980). Hexane-treated flies were transferred to $25^{\circ} \mathrm{C}$ and sampled 24,48 or $72 \mathrm{~h}$ later.

To generate flies expressing the maternal effect, pupae that had been in diapause were used to start the next generation of adults. Hexane-treated flies were maintained at $25^{\circ} \mathrm{C}$ until adult emergence. Flies were then transferred to either short-day or long-day conditions. Larval 
progeny of females with a diapause history $(\mathrm{DH})$ were reared under the same light regime as their parents (short- or long-day, respectively).

\subsection{Histone H3 Total Acetylation}

Total acetylation of histones H3 was measured for histones extracted from diapausing or nondiapausing pupae and post-diapause pharate adults using kits from Abcam (Cambridge, MA, USA) according to the manufacturer's protocol. Absorbance was measured at $450 \mathrm{~nm}$ using a microplate reader (FLUOstar Omega, BMG Labtech, Ortenberg, Germany), and the total amount of acetylated histone $\mathrm{H} 3$ was calculated from a stand curve. Values were normalized per mg protein as measured using a Bradford protein assay kit also from Abcam.

\subsection{Transcript Profiling}

S. bullata homologs of Drosophila melanogaster genes that have a known role in histone acetylation or deacetylation, including histone deacetylases, histone acetytransferases, and acetyl-CoA generating enzymes were identified using a comparative approach (Reynolds et al., 2013). Briefly, nucleotide sequences of $D$. melanogaster genes were identified using Flybase (http://flybase.org/) and were used to perform tblastx searches (Altschul et al., 1990) against a draft S. bullata genome (Peyton and Denlinger, unpublished data). Primers were designed using PrimerQuest software (Integrated DNA Technologies, Coralville, IA, USA). Results from BLAST searches are listed in Table 1.

Quantitative RT-PCR (qRT-PCR) was used to assess relative transcript abundance in first instar larvae, the photosensitive stage when pupal diapause is programmed; phanerocephalic pupae, the stage of diapause in S. bullata; and post-diapause pharate adults that had been treated with hexane $24 \mathrm{~h}$ previously to terminate diapause. Flies were designated as $\mathrm{DH}$ if they were from mothers with a diapause history or NDH if produced by mothers with no diapause history. Total RNA was isolated with a protocol that combined TRIzol ${ }^{\circledR}$ with the Purelink RNA Isolation Kit (Life Technologies, Grand Island, NY USA). Purelink DNase (Life Technologies) was used to remove genomic DNA.

cDNA was synthesized using the iScript cDNA synthesis system (Bio-Rad Laboratories Inc., Hercules, CA, USA) according to the manufacturer's instructions. The concentration of total RNA in each sample was measured with a NanoDrop spectrophotometer (Thermo Scientific, 
Wilmington, DE, USA), and $1 \mu \mathrm{g}$ total RNA was used in each reaction. Two independent reactions were carried out for each replicate sample and then pooled.

The relative mRNA expression of selected genes of interest was measured using an $\mathrm{iQ5}^{\mathrm{TM}}$ Multicolor Real-time PCR Detection System (Bio-Rad) and iQ ${ }^{\text {TM }}$ SYBR Green Supermix (Bio-Rad) as previously described (Reynolds et al., 2013). Primer sequences, designed using PrimerQuest software (Integrated DNA Technology, Coralville, IA, USA), conformed to MIQE standards (Bustin et al., 2010) and are shown in Supplemental Table S1. Relative transcript abundance was calculated for replicate samples using a modified $2^{-\Delta \mathrm{Ct}}$ as previously described (Reynolds et al., 2013). The geometric mean of $C_{t}$ values measured for $r p 49$ and $r p / 11$ was used as a normalizer.

\subsection{HDAC Activity}

Enzyme activity of HDACs that are sensitive to the HDAC inhibitor Trichostatin A (TSA), namely HDACs 1, 4, and 6, was measured in nuclear extracts prepared from non-diapause, diapause, and post-diapause pupae using a NE-PER ${ }^{\mathrm{TM}}$ Nuclear and Cytoplasmic Extraction Kit (Pierce Rockford, IL, USA) according to the manufacturer's instructions. Nuclear fractions were stored at $-80^{\circ} \mathrm{C}$ until used for the assays. HDAC enzyme activity was measured using an assay kit from Cayman Chemicals (Ann Arbor, MI, USA) according the manufacturer's protocol, except that the assay was performed at $25^{\circ} \mathrm{C}$ and ran for $60 \mathrm{~min}$. Fluorescence was measured with a microplate reader (FLUOstar Omega, Excitations $=340 \mathrm{~nm}$, Emission $=440 \mathrm{~nm}$ ). The amount of acetylated product was calculated from a standard curve. Values were normalized per $\mu \mathrm{g}$ protein and presented as $\mu \mathrm{M}$ acetylated product per min per $\mu \mathrm{g}$ protein.

\subsection{Statistical Analyses}

All statistical analyses were performed using MiniTab software (MiniTab, State College, PA, USA). Comparisons of histone H3 acetylation (Fig. 1), transcript abundance (Figs. 2, 3, 6, and 8), and HDAC activity (Fig. 7) for nondiapause, diapause, and post-diapause flies were assessed with One-way ANOVA followed by Fisher's LSD for post hoc pair-wise comparisons. Student's t-test was used to compare relative expression for photosensitive first instars (Figs. 4 and 5) comparisons. P-values from Student's t-tests were corrected with False Discovery Rate analyses (FDR, Benjamini and Hochberg, 1995) to reduce the probability of type I errors due to 
multiple comparison testing. For illustration purposes, bar graphs of relative transcript abundances are shown as normalized values, but all statistical values were performed using raw, non-normalized data.

\section{Results}

\subsection{Differences in total acetylation of histones H3.}

Total acetylation of histone $\mathrm{H} 3$ was reduced by at least $70 \%$ in early diapause pupae compared to the other groups measured (Fig. 1). Histone H3 acetylation increased as diapause progressed, and late diapause pupae had the same amount as nondiapause pupae. Histone H3 acetylation increased further after applying hexane to pupae to terminate diapause. Seventytwo hours post-diapause termination $\mathrm{H} 3$ acetylation was 2-fold higher than acetylation measured in late diapause or non-diapause pupae.

\subsection{Differences in mRNA expression of HATs, HDACs, and accessory genes}

\subsubsection{Identification of S. bullata HAT, HDAC, and accessory gene sequences}

Twenty-three genes encoding putative histone acetyltransferases and 10 genes that encode histone deacetylases have been identified in D. melanogaster (Feller et al. 2015). Homologs of many of these genes are also present in the S. bullata genome (Peyton and Denlinger, unpublished data), including three HAT encoding genes, tip60, p300, and gcn5 (Table 1) and nine HDAC-encoding genes, rpd3/hdac1, hdac3, hdac4, hdac6, sirt1/sir2, sirt2, sirt4, sirt6, and sirt7. We also identified S. bullata homologs of reptin, a component of the Tip60 histone acetyltransferase complex, as well as Acetyl-CoA carboxylase (acc) and ATP citrate lyase (acly), enzymes required to generate the pool of acetyl-CoA that serves as the acetyl group donor for histone acetylation.

\subsubsection{Changes in MRNA expression of HATs and HDACs}

Two of three HAT-encoding genes (Fig. 2) were differentially expressed in diapausing $S$. bullata pupae compared to their nondiapausing counterparts. Gcn5 transcription was downregulated during diapause by approximately $20 \%$. Transcription of tip60 was elevated approximately 2 -fold during diapause. reptin, a Tip60 complex component, was also elevated. This gene was 1.2-fold more abundant in early diapause pupae and 1.7-fold higher in late 
diapause pupae compared to nondiapause flies. Transcription of $p 300$ was the same in diapausing and nondiapausing flies.

Five HDAC genes were differentially expressed during diapause (Fig. 3). Transcription of hdac 3 and sirt 1 was reduced by $50 \%$ in early and late diapause, and sirt 2 mRNA abundance was reduced by $\sim 20 \%$. Sirt 4 transcription remained unchanged in early diapause but increased a moderate, but significant, $20 \%$ in late diapause. Similarly, sirt7 mRNA expression in early diapausing pupae was the same as that of nondiapausing pupae, but transcription of this gene was downregulated as diapause progressed. Sirt6 mRNA expression was the same in nondiapause and diapause flies. There were also no differences in $r p d 3 / h d a c 1$ or hdac4 transcription in diapausing flies.

\subsection{3 mRNA expression differences related to diapause termination}

Diapause in S. bullata can be broken with a topical application of hexane, and within 24 $\mathrm{h}$ after applying hexane to the heads of late diapause pupae there were significant changes in transcription levels of all HAT genes measured (Fig. 2) and in 6 HDAC genes (Fig. 3). Gcn5 and p300 were upregulated 1.5-fold. Tip60 was downregulated by $50 \%$, and reptin was downregulated $\sim 20 \%$. The change in Hdac3 expression was the most profound, with a 3-fold increase in abundance after hexane application. Hdac6, sirt6, and sirt7 were each upregulated 1.8-fold, and sirt2 increased 1.4-fold. Sirt4 was downregulated 40\%. Rpd3/hdac1, hdac4, and sirt1 did not change in response to hexane. It is notable that post-diapause, the amount of mRNA for the majority of genes was nearly the same as that measured for nondiapause pupae. One exception was sirt1 which was 50 \% less abundant in post-diapause flies than in nondiapause pupae.

\subsubsection{Differences in $m R N A$ expression related to diapause programming}

In S. bullata, the programming of pupal diapause is initiated in photosensitive embryos and first-instar larvae that are reared under a short-day photoperiod (Denlinger, 1971). We compared transcript abundances of four HDAC-encoding genes, rpd3/hdac1, hdac3, hdac6, and sirt2 in first-instar larvae reared in diapause-inducing, short-day conditions compared to firstinstar larvae reared in diapause-averting, long-day conditions. All four of these genes were significantly more abundant in first-instar larvae that were programmed to enter diapause (Fig. 
4). Hdac1 transcription was 2 -fold higher in pre-diapause larvae $(P<0.0001)$, hdac3 was 1.5 fold higher $(\mathrm{P}=0.0008)$, and both hdac6 and sirt2 were approximately 1.8 -fold more abundant $(P=0.002$ for both genes).

\subsubsection{Differences in $m R N A$ expression related to a maternal effect that prevents diapause}

In S. bullata the mother's diapause history influences her progeny's potential for diapause. Flies from females that experienced pupal diapause are unable to enter diapause even when reared in strong, diapause-inducing conditions. This set of experiments examined the impact of the mother's diapause history on the expression of genes that encode selected HDACs: rpd3/hdac1, hdac3, hdac6, and sirt2 in photosensitive first-instar larvae and in pupae that were produced by either mothers with a diapause history $(\mathrm{DH})$ or with no diapause history (NDH). Flies from both types of mothers were reared under either a short-day (SD) or long-day (LD) photoperiod to give 4 types of flies: DH-SD, DH-LD, NDH-SD, or NDH-LD. Only NDH-SD flies can enter diapause.

Expression of the maternal effect can be seen early in development: significant differences in the mRNA profiles of HDAC-encoding genes $r p d 3 / h d a c 1, h d a c 3$, hdac6, and sirt2 were evident in first-instar larvae that were reared under a short-day photoperiod (Fig. 5A). First-instar larvae from mothers with no diapause history had $60 \%$ more rpd3/hdac1 mRNA (P $=0.022$ ) and $65 \%$ more hdac3 and hdac6 mRNA ( $P=0.022$ for both genes). Sirt 2 transcription was $\sim 40 \%$ higher in NHD-SD larvae compared to DH-SD larvae $(P=0.008)$. There were no significant differences between NHD and DH first-instar larvae that were reared under long-day, diapause-averting conditions (Fig. 5B). It was not possible to directly compare NDH-LD and DHLD larvae to DH-SD larvae because minor differences in rearing temperature resulted in longday reared larvae that were slightly more mature than short-day reared larvae on the day of collection.

At the pupal stage, transcription of hdac-encoding genes was lower in diapausing flies compared to nondiapausing pupae, regardless of whether they failed to enter diapause because of their long-day rearing conditions or could not enter diapause because of their mother's diapause history (Fig. 6). There were no differences among the 3 nondiapause types (i.e., NDH-LD, DH-SD and DH-LD) for most of the hdacs measured. Only hdac6 was differentially 
expressed among the three nondiapause groups, with more mRNA in DH-SD flies than DH-LD flies.

\subsection{Differences in HDAC activity related to diapause}

We found significant differences in the enzyme activity of TSA sensitive HDACs (i.e. HDAC1, HDAC3, and HDAC6) in diapausing pupae compared to nondiapausing and post-diapausing pupae (ANOVA, $F=6.91 ; \mathrm{P}=0.001$; Fig. 7). HDAC activity was reduced by $30 \%$ in nuclear extracts prepared from diapausing pupae, compared to extracts from nondiapausing pupae (Fisher's LSD P < 0.05). HDAC activity remained low $24 \mathrm{~h}$ after hexane was applied to terminate diapause and was not significantly different than activity in diapausing pupae, but by $48 \mathrm{~h}$ posthexane application, HDAC activity rose significantly and was equivalent to that observed in nondiapausing pupae.

\subsection{Diapause-related differences in genes involved in acetyl-CoA production and metabolism}

Histone acetylation depends on the pool of acetyl-CoA available to donate acetyl groups (Takahashi et al., 2006). Transcription of genes encoding ATP-citrate lyase (acly) and Acetyl-CoA carboxylase (acc), two genes necessary to generate the acetyl-CoA pool (Galdieri and Vancura, 2012; Wellen et al., 2009), were both significantly downregulated during diapause (Fig. 8). Acc transcription was reduced by $50 \%$ in both early and late diapause. Acly mRNA expression was $70 \%$ lower in diapausing pupae compared to nondiapausing pupae.

\section{Discussion}

The results reported here support our hypothesis that changes in histone acetylation are associated with the manifestation of pupal diapause in S. bullata. First, we have shown that total acetylation of histone $\mathrm{H} 3$ is reduced $70 \%$ in early diapause compared to same-stage, nondiapause pupae. We also showed a 20 \% reduction in transcription of gcn5, a HAT-encoding gene that may be responsible for the reduction in histone H3 acetylation. Other diapauseassociated changes in genes and enzymes associated with histone acetylation/deacetylation 
include an up to 2 -fold increase in $h d a c 1, h d a c 3, h d a c 6$, and sirt2 mRNA in diapauseprogrammed first-instar larvae; a 1.8-fold increase in transcription of tip60 and reptin, genes encoding parts of the Tip60 HAT complex gene; a substantial $40-60 \%$ downregulation of hdac3 and sitr1 in diapausing pupae; and a $30 \%$ reduction in TSA-sensitive HDAC activity during diapause. Taken together these results clearly show that changes in the amount of histone H3 acetylation and shifts in HAT and HDAC abundance and activity are associated with diapause in this species, and we predict that these changes have a significant impact on the programming, maintenance and termination of diapause as discussed below.

The current paradigm suggests that adding acetyl groups to histone proteins activates gene expression while removing acetyl groups represses transcription. Given that $S$. bullata pupal diapause is characterized by large-scale downregulation of transcription (Denlinger, 2002), we think it likely that the reduced histone H3 acetylation observed in early diapause contributes to gene silencing. Our prediction is consistent with previously published studies on histone acetylation in hibernating mammals. In thirteen-lined ground squirrels, histone H3 acetylation was reduced by $50 \%$ when animals entered torpor which, the authors conclude, halts transcription (Biggar and Storey 2014; Morin and Storey, 2009). We predict that a similar mechanism may be operating in diapausing pupae of $S$. bullata, but further research is needed to confirm a link between the level of histone $\mathrm{H} 3$ acetylation and the transcription rate during diapause in these flies.

Reduced histone acetylation in diapausing $S$. bullata is likely coordinated between changes in both HAT and HDAC amount and activity, thus we expected to find a decrease in HAT-gene transcription and an increase in HDAC-transcription in diapausing pupae compared to their nondiapause counterparts. Of the HAT-encoding genes measured only gcn 5 mRNA expression decreased as expected with a $20 \%$ decrease in mRNA abundance in both early and late diapause pupae. Other HAT genes measured either increased (tip60) or remained unchanged ( $p 300$ ). One possible explanation for this is that tip60 and p300 primarily acetylate histone $\mathrm{H} 4$, which we were unable to measure because of technical problems associated with the use of an assay designed for mammals. In D. melanogaster, Tip60 can generate silent 
chromatin through increased acetylation of histone H4 proteins (Qi et al. 2006), and it is possible this function of Tip60 has been conserved in S. bullata.

Our finding that HDAC-gene transcription and enzyme activity were reduced in diapausing pupae was especially unexpected given that decreased histone acetylation is commonly associated with an increase in HDAC amount and activity, while knockdown in hdac expression is associated with increased acetylation. Indeed, knockdown of any one of the HDAC-encoding genes in D. melanogaster cells is sufficient to increase global histone acetylation (Feller et al., 2015). Similarly, we were surprised to find increased transcription of HDAC genes in post-diapause pharate adults, which had increased levels of acetylated histone $\mathrm{H} 3$, and in photosensitive first-instar larvae that were programmed for diapause: both of these stages could be expected to be developmental periods with high rates of transcription.

One possible explanation for these seemingly contradictory results is that changes in histone acetylation may take place before the pupae enter diapause. Histone deacetylation in photosensitive first-instar larvae, for example, may be necessary to remove acetylation marks that promote direct development and to initiate changes in gene expression that are needed to successfully switch from a program of direct development to a developmental pathway that includes diapause. Indeed, transcription of these genes was significantly less in first-instar larvae that were not programmed to enter diapause either because they were reared in diapause-averting, long-day conditions or because the maternal block of diapause in this species prevents diapause programming. Additional testing of HDAC activity and histone acetylation in first-instar larvae that are programmed to enter diapause is necessary to completely test these hypotheses.

Another explanation for the apparent mismatch between HDAC activity and amount of histone $\mathrm{H} 3$ acetylation is that factors other than HAT and HDAC activity may mediate the level of histone acetylation during diapause. A reduced pool of acetyl-CoA, which serves as a donor of acetyl groups, may be one such limiting factor in diapausing pupae (Takahashi at al., 2006). Previous research on S. crassipalpis showed significantly reduced levels of citrate, a precursor to acetyl-CoA generation, in diapausing pupae (Michaud and Denlinger, 2007), and that could potentially limit acetyl-CoA availability. In addition, our current study showed that genes 
encoding ATP-citrate lyase (ACLY) and Acetyl-CoA carboxylase (ACC), both key influencers of histone acetylation (Peleg et al., 2016; Chypre et al., 2012; Galdieri and Vancura, 2012, Wellen et al., 2009), were substantially downregulated during diapause. Future studies will allow us to determine whether downregulation of these genes causes or simply correlates with diapause entry in S. bullata and whether limited availability of acetyl-CoA prevents histone acetylation during diapause.

It is also possible that the HDAC function in diapause is not limited to activation or silencing of genes but that HDAC inhibition (i.e., downregulation) drives a number of biological processes (e.g. cell-cycle progression, metabolism, and stress-response) that are altered during diapause. For example, chemical inhibition of HDAC3 represses key metabolic genes including hexokinase, fatty acid synthase, glyceride-3-phosphate, and cytochrome c oxidase subunit Vib (Kant et al., 2002). Thus, HDAC inhibition may be linked to the metabolic depression that is a defining characteristic of insect diapause, including that of S. bullata (Denlinger et al., 1972).

HDAC inhibition could also mediate diapause-related environmental stress responses, especially the increased production of heat shock proteins (Hsps), which is another common feature of diapause. Diapause upregulation of Hsps is wide-spread in insects, including flesh flies (Zhang and Denlinger, 2010; Rinehart et al., 2007). Expression of Hsp-coding genes is negatively correlated with HDAC activity in D. melanogaster (Westerheide et al., 2009; Zhao et al., 2005a; Zhao et al., 2005b; Kang et al., 2002). Both basal and heat-induced expression of hsp70 increase in larvae treated with HDAC inhibiting chemicals (Zhao et al., 2007), and transcription of $h s p 22$ (Tao et al, 2004), hsp26 (Nightingale et al., 1998), hsp60, and dnaj/hsp40 (Kang et al., 2002) increases in response to HDAC3 knockdown. Future studies with S. bullata will assess the effect of HDAC knockdown on the ability of these flies to resist environmental stress.

Although the precise role of histone acetylation and/or deacetylation in the diapause of S. bullata remains unknown, the clear and consistent results we obtained suggest that these post-translational events are critically involved in the diapause of S. bullata, and possibly in other insects as well. This, along with evidence that small RNAs contribute to diapause regulation (Reynolds et al., 2013), adds a putative epigenetic level of control that likely presides 
over the well-documented environmental and hormonal control mechanisms that have been known to regulate insect diapause. A comprehensive model for understanding diapause regulation will need to incorporate these new dimensions as well.

Acknowledgements: We thank members of the Denlinger lab for helpful suggestions during the preparation of this manuscript. This work was supported in part by National Science Foundation Grant Foundation Grant IOS-1354377. 


\section{References}

Altschul, S.F., Gish, W., Miller, W., Myers, E.W., Lipman, D.J., 1990. Basic local alignment search tool. J Mol Biol, 215: 403-410.

Amsalem, E., Galbraith, D.G., Cnaani, J., Teal, P.E.A., Grozinger, C.M. 2015. Conservation and modification of genetic and physiological toolkits underpinning diapause in bumble bee queens. Mol Ecol, 24: 5596-5615.

Benjamini, Y., Hochberg, Y., 1995. Controlling the false discovery rate: a practical and powerful approach to multiple testing. J R Stat Soc Ser B Stat Methodol, 57: 289-300.

Biggar, Y and Storey, K.B. 2014. Global DNA modifications suppress transcription in brown adipose tissue during hibernation.Cryobiology 333-338.

Bustin, S, Beaulieu, J.-F., Huggett, J., Jaggi, R, Kibenge, F.S.B., Olsvik, P.A., Penning, L.C., Toegel, S. 2010. MIQE précis: Practical implementation of minimum standard guidelines for fluorescence-based quantitative real-time PCR experiments. BMC Mol Biol, 11: 74.

Byron, N. Wybouw, W. Dermauw, L. Tirry, T. van Leeuwen, 2013, Genome wide geneexpression analysis of facultative reproductive diapause in the two-spotted spider mite Tetranychus urticae. BMC Genomics 14:815.

Chypre, M., Zaidi, N., and Smans, K. 2012. ATP-citrate lyase: a mini-review. Biochem. Biophys. Res. Commun., 422:1-4.

Cosentino, C., Mostoslavsky, R. 2013. Metabolism, longevity and epigenetics. Cell Mol Life Sci, 70: 1525-1541.

Denlinger, D.L., 2002. Regulation of diapause. Annu Rev Entomol, 47: 93-122. 
Denlinger, D.L., 1972. Induction and termination of pupal diapause in Sarcophaga (Diptera: Sarcophagidae). Biol Bull, 142: 11-24.

Denlinger, D.L., 1971. Embryonic determination of pupal diapause in the flesh fly Sarcophaga crassipalpis Macquart. J Insect Physiol 17, 1815-1822.

Denlinger, D.L., Campbell, J.J., Bradfield, J.Y., 1980. Stimulatory effect of organic solvents on initiating development in diapausing pupae of the flesh fly Sarcophaga crassipalpis and the tobacco hornworm Manduca sexta. Physiol Entomol 5, 7-15.

Denlinger, D.L., Willis, J.H., Fraenkel, G., 1972. Rates and cycles of oxygen consumption during diapause in Sarcophaga flesh flies. J Insect Physiol, 871-882.

Fogg, P.C.M., O’Neill, J.S., Dobrzycki, T., Calvert, S., Lord, E.C., McIntosh, R.L.L., Elliot, C.J.H., Sweeney, S.T., Hastings, M.H., Chawla, S. 2014. Class Ila histone deacetylases are conserved regulators of circadian function. J Biol Chem, 289: 34341-34348.

Furusawa, C., Kaneko, K. 2013. Epigenetic feedback regulation accelerates adaptation and evolution. PLOS ONE 8(5): e61251.

Galdieri,L., Vancura, A. 2012. Acetyl-CoA carboxylase regulates global histone acetylation. J Biol Chem, 287: 23865-23876.

Hahn, D.A., Ragland, G.J., Shoemaker, D.D., Denlinger, D.L., 2009. Gene discovery using massively parallel pyrosequencing to develop ESTs for the flesh fly, Sarcophaga crassipalpis. BMC Genomics 10, 234.

Henrich, V.C., Denlinger, D.L., 1982. A maternal effect that eliminates pupal diapause 
in progeny of the flesh fly Sarcophaga bullata. J Insect Physiol 28, 881-884.

Jaenisch, R., Bird, A. 2003. Epigenetic regulation of gene expression: how the genome integrates intrinsic and environmental signals. Nature Genet, 33: 245-254.

Kamemura, K., Ogawa, M., Ohkubo, S., Ohtsuka, Y., Shitara, Y., Komiya, T., Maeda, S., Ito, A., Yoshida, M. 2012. Depression of mitochondrial metabolism by downregulation of cytoplasmic deacetylase, HDAC6. FEBS Lett, 586: 1379-1383.

Kang, H., Benzer, S., Min, K.-T., 2002. Life extension in Drosophila by feeding a drug. Proc NatI Acad Sci, USA, 99: 838-843.

Karpac, J., Jasper, H.2011. Metabolic homeostasis: HDACs take center stage. Cell, 145:497-499.

Katada, S., Imhof, A, Sassone-Corsi, P. 2012. Connecting threads: epigenetics and metabolism. Cell, 148: 24-28.

Kilvitis, H.J., Alvarez, M., Foust, C.M., Schrey, A.W., Robertson, M., Richards, C.L. 2014. Ecological epigenetics. C.R. Landry and N. Aubin-Horth (eds.), Ecological Genomics: Ecology and the Evolution of Genes and Genomes, Advances in Experimental Medicine and Biology 781, DOI 10.1007/978-94-007-7347-9_10.

Kubrak, O.I., Kucerova, L., Theopold, U., Nassel, D.R. 2014. The sleeping beauty: how reproductive diapause affects hormone signaling, metabolism, immune response and somatic maintenance in Drosophila melanogaster. PLoS ONE 9(11): e113051.

Lu, Y.X., Denlinger, D.L., Xu, W.H. 2013. Polycomb repressive complex 2 (PRC2) protein ESC regulates insect developmental timing by mediating H3K27me3 and activating prothoracicotropic hormone gene expression. J Biol Chem, 288:23554-64. 
Malapeira, J., Khaitova, L.C., Mas, P. 2012. Ordered changes in histone modifications at the core of the Arabidopsis circadian clock. Proc Natl Acad Sci, USA, 109: 21540-21545.

Matthias, P., Yoshida, M. Khochbin, S. 2008. HDAC6 a new cellular stress surveillance factor. Cell Cycle, 7: 7-10.

Morin, P., Jr., Storey, K.B. 2006. Evidence for a reduced transcriptional state during hibernation in ground squirrels. Cryobiology, 53: 310-318.

Michaud, M.R., Denlinger, D.L., 2007. Shifts in the carbohydrate, polyol, and amino acid pools during rapid cold-hardening and diapause-associated cold-hardening in flesh flies (Sarcophaga crassipalpis): a metabolomic comparison. J Comp Physiol, 177B: 753-763

Nightingale, K.P., Wellinger, R.E., Sogo, J.M., Becker, P.B. 1998. Histone acetylation facilitates RNA polymerase II transcription of the Drosophila hsp26 gene in chromatin. EMBO J, 17: 28652876.

Peleg, S., Feller, C., Forne, I., Schiller, E., Sévins, D.C., Shauer, T., Regnard, C., Straubz, T., Prestel, M., Klima, C., Nogueira, M.S., Becker, L., Klopstock, T., Sauer, U., Becker, P.B., Imhof, A., Ladurner, A.G. 2016. Life span extension by targeting a link between metabolism and histone acetylation in Drosophila. EMBO Reports, 17: 455-469.

Poelchau, M.F., Reynolds, J.A., Elsik, C.G., Denlinger, D.L., Armbruster, P.A. 2013. RNA-Seq reveals early distinctions and late convergence of gene expression between diapause and quiescence in the Asian tiger mosquito, Aedes albopictus. J Exp Biol, 216: 4082-4090.

Ragland, G.J., Denlinger, D.L., Hahn, D.A., 2010. Mechanisms of suspended animation are revealed by transcript profiling in the flesh fly. Proc Natl Acad Sci, USA, 107: 14909-14914. 
Ragland, G.J., Egan, S.P., Feder, J.L., Berlocher, S.H., Hahn, D.A. 2011. Developmental trajectories of gene expression reveal candidates for diapause termination: a key life-history transition in the apple maggot fly Rhagoletis pomonella. J Exp Biol, 214: 3948-3959.

Reynolds J. A., Clark J., Diakoff S.J., Denlinger D.L. 2013. Transcriptional evidence for small RNA regulation of pupal diapause in the flesh fly, Sarcophaga bullata. Insect Biochem Mol Biol, 43:982-9.

Reynolds, J.A., Poelchau, M.F., Rahman, Z., Armbruster, P.A., Denlinger, D.L. 2012. Transcript profiling reveals mechanisms for lipid conservation during diapause in the mosquito, Aedes albopictus. J Insect Physiol, 58: 966-73.

Reynolds, J.A. and Hand, S.C. 2009. Embryonic diapause highlighted by differential expression of mRNAs for ecdysteroidogenesis, transcription and lipid sparing in the cricket Allonemobius socius. J Exp Biol, 212: 2075-84.

Rinehart, J.P., Robich, R.M., Denlinger, D.L., 2010. Isolation of diapause-regulated genes from the flesh fly, Sarcophaga crassipalpis by suppressive subtractive hybridization. J Insect Physiol, 56: 603-609.

Rinehart, J.P., Li, A., Yocum, G.D., Robich, R.M., Hayward, S.A., Denlinger, D.L., 2007. Upregulation of heat shock proteins is essential for cold survival during insect diapause. Proc Natl Acad Sci, USA, 104: 11130-11137.

Rockey, S.J., Miller, B.B., Denlinger, D.L., 1989. A diapause maternal effect in the flesh fly, Sarcophaga bullata: transfer of information from mother to progeny. $J$ Insect Physiol, 35: 553-558. 
Sim, C., Denlinger D. L. 2009. Transcription profiling and regulation of fat metabolism genes in diapausing adults of the mosquito Culex pipiens. Physiol Genomics, 39: 202-209.

Svetec, N., Pavlidis, P., Stephan, W. 2009. Recent strong positive selection on Drosophila melanogaster HDAC6, a gene encoding a stress surveillance factor, as revealed by population genomic analysis. Mol Biol Evol, 26:1549-1556.

Takahashi, H., McCaffery, J. M., Irizarry, R. A., and Boeke, J. D. (2006) Nucleocytosolic acetylcoenzyme A synthetase is required for histone acetylation and global transcription. Mol. Cell 23, 207-217

Tao, D., Lu, J., Sun, H., Zhao, Y.-M., Yuan, Z.-G., Li, X.-X., Huang, B.-I. 2004. Trichotatin A extends the lifespan of Drosophila melanogaster by elevating hsp22 expression. Acta Biochim Biophys Sin, 36: 618-622.

Toni, L.S., Padilla, P.A. 2016. Developmentally arrested Austrofundulus limnaeus embryos have changes inpost-translational modifications of histone H3. J. Exp. Biol. 219: 544-552.

Tormey, D., Colbourne, J.K., Mockaitis, K., Choi, J.-H., Lopez, J., Burkhart, J., Bradshaw, W., Holzapfel, C., 2015. Evolutionary divergence of core and post-translational circadian clock genes in the pitcher-plant mosquito, Wyeomyia smithii. BMC Genomics, 16: 754.

Wadsworth, C.B., Dopman, E.B. 2015. Transcriptome profiling reveals mechanisms for the evolution of insect seasonality. J Exp Biol, doi: 10.1242/jeb.126136.

Wellen, K.E., Hatzivassiliou, G., Sachdeva, U.M., Bui, T.V., Cross, J.R., Thompson, C.B. 2009. ATPcitrate lyase links cellular metabolism to histone acetylation. Science, 324: 1076-1080. 
Westerheide, S.D., Anckar, J., Stevens, S.M., Jr., Sistonen, L., Morimoto, R.I. 2009. Stressinducible regulation of Heat Shock Factor 1 by the deacetylase SIRT1. Science, 323: 1063-1066.

Yamaguchi, T, Cubizolles, F., Zhang, Y., Reichert, N., Kohler, H., Seiser, C. and Matthias, P. 2010. Histone deacetylases 1 and 2 act in concert to promote the G1-to-S progression. Genes Dev, 24: 455-469.

Yocum, G.D., Rinehart, J.P., Horvath, D.P., Kemp, W.P., Bosch, J. Alroobi, R., Salem, S. 2015. Key molecular processes of the diapause to post-diapause quiescence transition in the alfalfa leafcutting bee Megachile rotundata identified by comparative transcriptome analysis. Physiol Entomol, 40: 103-112.

Zhang, Q and Denlinger, D.L. 2010 Molecular characterization of heat shock protein 90, 70 and 70 cognate cDNAs and their expression patterns during thermal stress and pupal diapause in the corn earworm. J Insect Physiol, 56:138-50.

Zhao, Y., Lu, J., Sun, H., Chen, X., Huang, W., Tao, D., Huang, B. 2005a. Histone acetylation regulates both transcription initiation and elongation of the hsp22 gene in Drosophila. Biochem Biophys Res Commun, 326: 811-816.

Zhao, Y., Sun, H., Lu, J. Li, X., Chen, X., Tao, D., Huang, W. Huang, B. 2005b. Lifespan extension and elevated hsp gene expression in Drosophila caused by histone deacetylase inhibitors. J Exp Biol, 208: 697-705.

Zhao, Y., Chen, X., Sun, H. Yuan, Z., Ren, G., Li, X., Lu, J., Huang, B. 2006. Effect of histone deacetylase inhibitors on hsp70 transcription regulation in Drosophila. Cell Res, 16: 566-576.

Zhao, Y., Lu, J., Sun, H., Chen, X., Huang, B. 2007. Roles of histone acetylation modification in basal and inducible expression of hsp26 gene in D. melanogaster. Mol Cell Bio, 306: 1-8. 
Zhou, R., Yang, F., Chen, D.-F., Sun, Y.-X., Yang, J.-S., Yang, W.-J. 2013. Acetylation of chromatinassociated histone H3 lysine 56 inhibits the development of encysted Artemia embryos. PLOS ONE, 8: e68374. 


\section{Figure Captions}

Figure 1. Total acetylation of histone H3 in nondiapause (ND), early diapause (ED), late diapause (LD and post-diapause (PD) pupae. ED and LD pupae had been in diapause for $15 \mathrm{~d}$ and $30 \mathrm{~d}$, respectively. PD pupae were sampled 48 and $72 \mathrm{~h}$ after treatment with hexane to terminate diapause. Amount of histone acetylation was normalized to $\mu \mathrm{g}$ per mg protein. Bars represent the mean \pm s.d. normalized to the non-diapause group, and bars labeled with different letters had significantly different means $(P<0.05) . N=5$ biological replicates.

Figure 2. mRNA abundance of select histone acetyltransferase (HAT) encoding genes pupae not in diapause (ND), early diapause (ED), late diapause (LD) or post-diapause (PD). ED and LD pupae had been in diapause for $15 \mathrm{~d}$ and $30 \mathrm{~d}$, respectively. PD pupae were sampled $24 \mathrm{~h}$ after treatment with hexane to terminate diapause. Bars represent the mean \pm s.d. normalized to the non-diapause group. For each gene, bars labeled with different letters had significantly different means. $\mathrm{N}=6-7$ replicates.

Figure 3. mRNA abundance of select histone deacetylase (HDAC) encoding genes in nondiapause (ND), early diapause (ED), and late (diapause) and in post-diapause pharate adults. ED and LD pupae had been in diapause for $15 \mathrm{~d}$ and $30 \mathrm{~d}$, respectively. PD pupae were sampled $24 \mathrm{~h}$ after treatment with hexane to terminate diapause. Bars represent the mean \pm s.d. normalized to the non-diapause group. For each gene, bars labeled with different letters had different means. $\mathrm{N}=6-7$ replicates.

Figure 4. mRNA abundance of select HDAC encoding genes in photosensitive first instar larvae programmed for diapause compared to first instar larvae not programmed for diapause. Both groups of larvae were from mothers with no diapause history and had the potential to enter diapause. Photosensitive larvae were programmed to enter diapause as pupae by rearing them under a short-day photoperiod; larvae not programmed to enter diapause as pupae were 
reared under long-day conditions. Bars represent the mean \pm s.d. normalized to the nondiapause group. $\mathrm{N}=4-6$ biological replicates.

Figure 5. Influence of maternal history on mRNA abundance of select HDAC encoding genes in photosensitive first instar larvae A) reared under short-day, diapause-inducing conditions or B) reared under long-day, diapause-averting conditions. Black bars indicate larvae from mothers with a diapause history; gray bars indicate mothers with no diapause history. Bars represent mean \pm s.d. normalized to the group with no diapause history. Pairs of bars labeled with "*” are significantly different from each other. $\mathrm{N}=4-6$ replicates.

Figure 6. mRNA expression profiles of candidate HDAC-encoding genes in diapausing (NDHSD) and nondiapause (NDH-LD, DH-SD, and DH-LD) pupae is influenced by maternal diapause history and rearing environment. NDH-SD pupae entered diapause since they were reared in a diapause-inducing environment and were from mothers with no diapause history. NDH-LD pupae were from mothers with no diapause history and did not enter diapause because they were reared in diapause-averting long day conditions. DH-SD and DH-LD pupae were from mothers that experienced diapause and thus could not enter diapause regardless of their rearing conditions. Bars represent the mean \pm s.d. normalized to the non-diapause group. For each gene, bars marked with different letters had significantly different means. $\mathrm{N}=4-6$ replicates.

Figure 7. HDAC activity in nondiapause, diapause, and post-diapause pupae. Nondiapause pupae were from mothers with no diapause history and were reared in diapause-averting conditions. Diapause pupae were from mothers with no diapause history and were reared in diapause-inducing conditions, and post-diapause pupae were generated by applying hexane to the heads of diapause pupae. Bars represent the mean \pm s.d. Bars marked with different letters had significantly different means. $\mathrm{N}=4-6$ replicates. 
Figure 8. mRNA expression profiles of acc and acly in nondiapause (ND), early diapause (ED) and late diapause (LD) pupae. ED and LD pupae had been in diapause for $15 \mathrm{~d}$ and $30 \mathrm{~d}$, respectively. Bars represent the mean \pm s.d. normalized to the non-diapause group. Bars labeled with different letters had significantly different means. $\mathrm{N}=6$ replicates. 
Table 1. Genes that encode putative HATs, HDACs and accessory proteins in S. bullata.

\begin{tabular}{|c|c|c|c|c|c|}
\hline Putative I.D. & Predicted & S. bullata genome I.D. & $\begin{array}{l}\text { Species with Closest } \\
\text { Identity }\end{array}$ & $\begin{array}{l}\mathrm{E}- \\
\text { value }\end{array}$ & $\begin{array}{l}\% \\
\text { I.D. }\end{array}$ \\
\hline$p 300$ & p300 & Sbullata.00006161-RA & M. domestica & 0.0 & $88 \%$ \\
\hline tip60 & Tip60 & Sbullata.00000684-RA & Stomoxys calcitrans & 0.0 & $89 \%$ \\
\hline reptin & Reptin & Sbullata.00007158-RA & D. melanogaster & 0.0 & $93 \%$ \\
\hline gen5 & GCN5 & Sbullata.00005213-RA & Musca domestica & 0.0 & $89 \%$ \\
\hline hdac1/Rpd3 & HDAC1 & Sbullata.00006253-RA & Stomoxys calcitrans & 0.0 & $98 \%$ \\
\hline hdac3 & HDAC3 & Sbullata.00002319-RA & Musca domestica & 0.0 & $94 \%$ \\
\hline hdac4 & HDAC4 & Sbullata.00003371-RA & Musca domestica & 0.0 & $86 \%$ \\
\hline hdac6 & HDAC6 & Sbullata.00005424-RA & Stomoxys calcitrans & 0.0 & $83 \%$ \\
\hline sirtuin 1 & Sirtuin1 & Sbullata.00000082-RA & Lucilia cuprina & 0.0 & $76 \%$ \\
\hline sirtuin2 & Sirtuin2 & Sbullata.00006172-RA & Lucilia cuprina & 0.0 & $85 \%$ \\
\hline sirtuin4 & Sirtuin4 & Sbullata.00005381-RA & S. calcitrans & 0.0 & $86 \%$ \\
\hline sirtuin6 & Sirtuin6 & Sbullata.00001382-RA & Lucilia cuprina & 0.0 & $92 \%$ \\
\hline sirtuin7 & Sirtuin7 & Sbullata.00003464-RA & Lucilia cuprina & 0.0 & $72 \%$ \\
\hline $\begin{array}{l}\text { acetyl-CoA } \\
\text { carboxylase }\end{array}$ & $\begin{array}{l}\text { Acetyl-CoA } \\
\text { Carboxylase }\end{array}$ & Sbullata.00000217-RA & S. calcitrans & 0.0 & $90 \%$ \\
\hline atp-citrate lyase & $\begin{array}{l}\text { ATP-citrate } \\
\text { lyase }\end{array}$ & Sbullata.00002173-RA & S. calcitrans & 0.0 & $92 \%$ \\
\hline
\end{tabular}




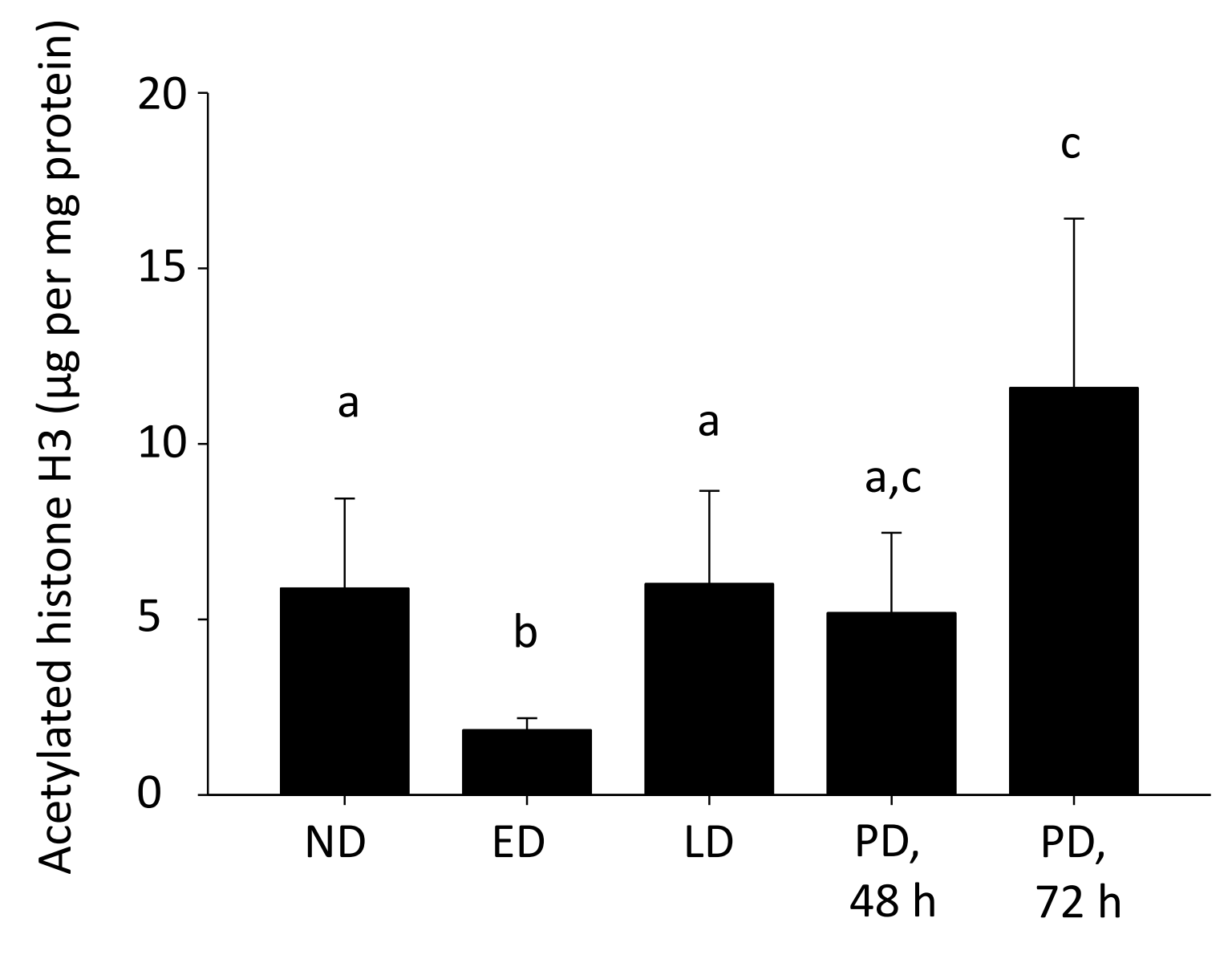

\footnotetext{
Figure 1

Figure 1
}

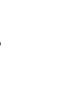

Figures

.

更

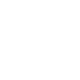

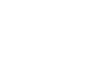
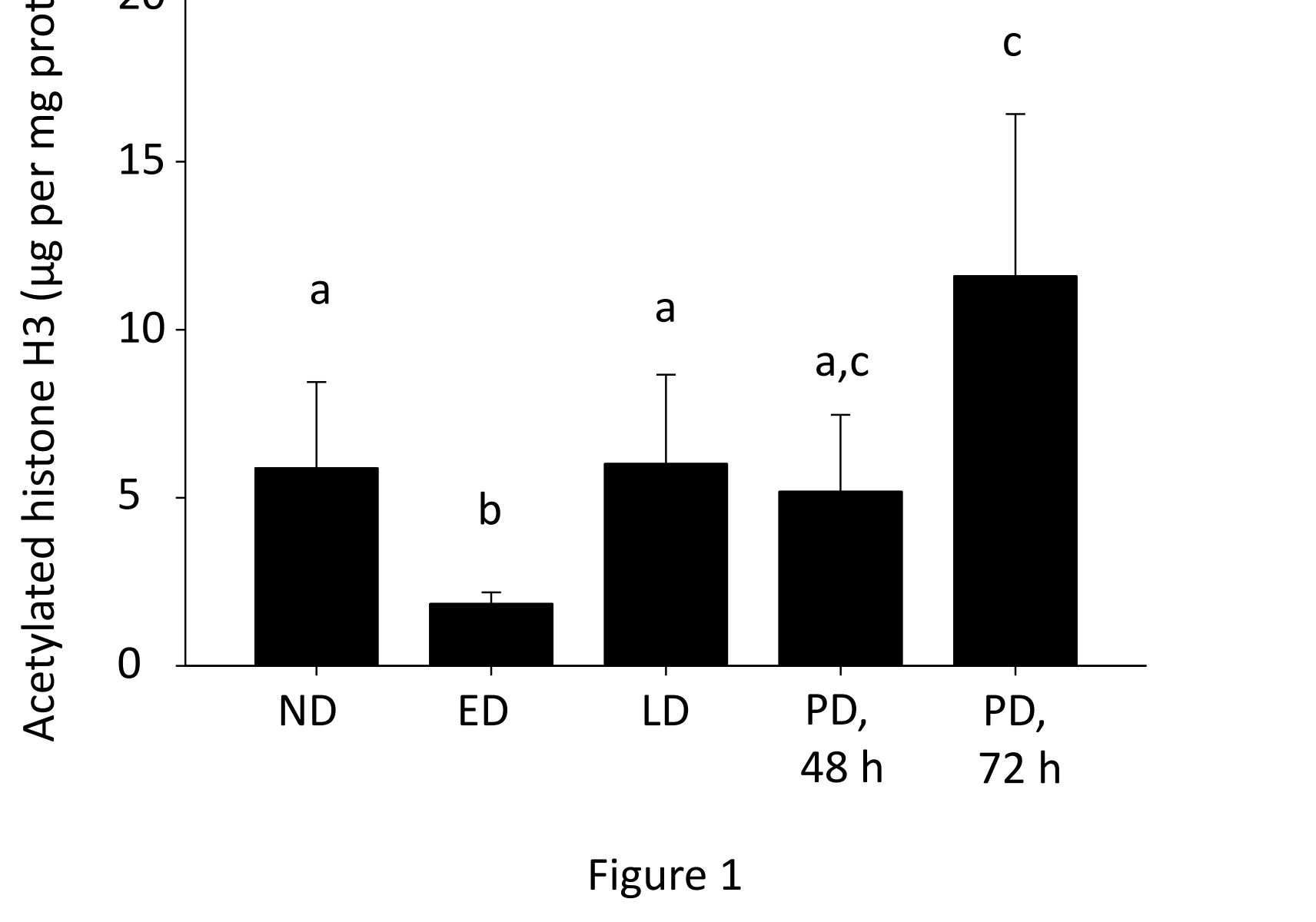


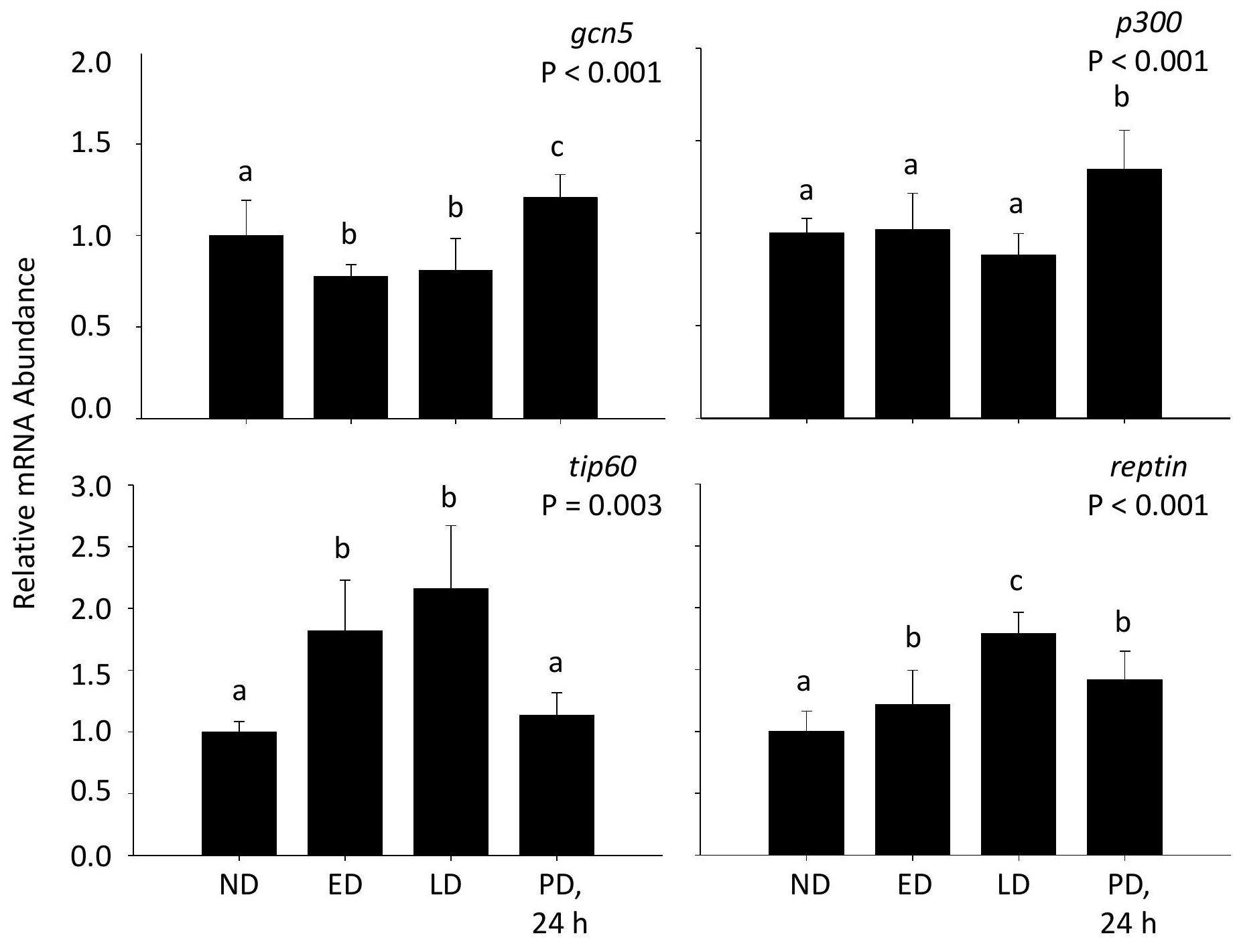

Figure 2 
hdac3
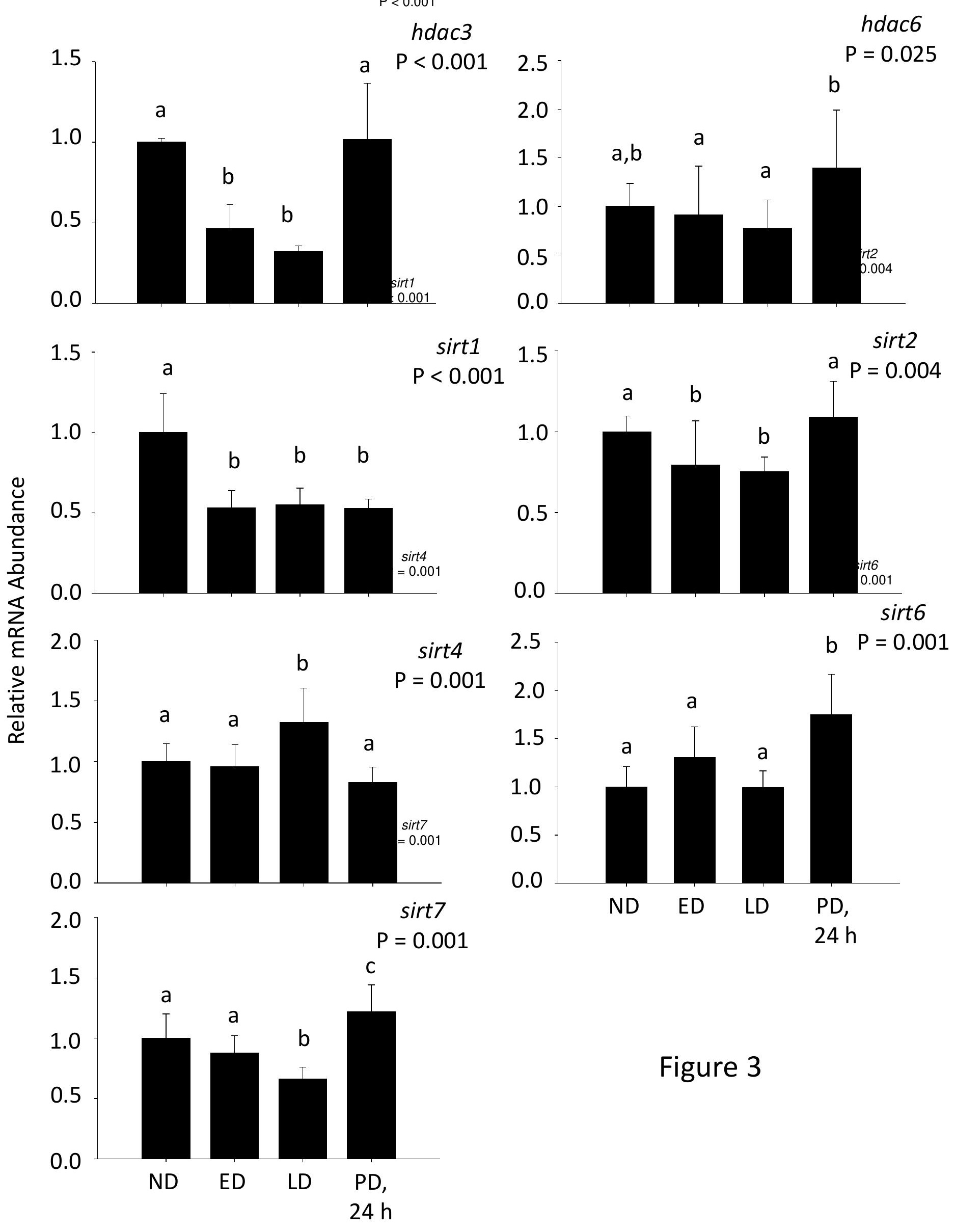


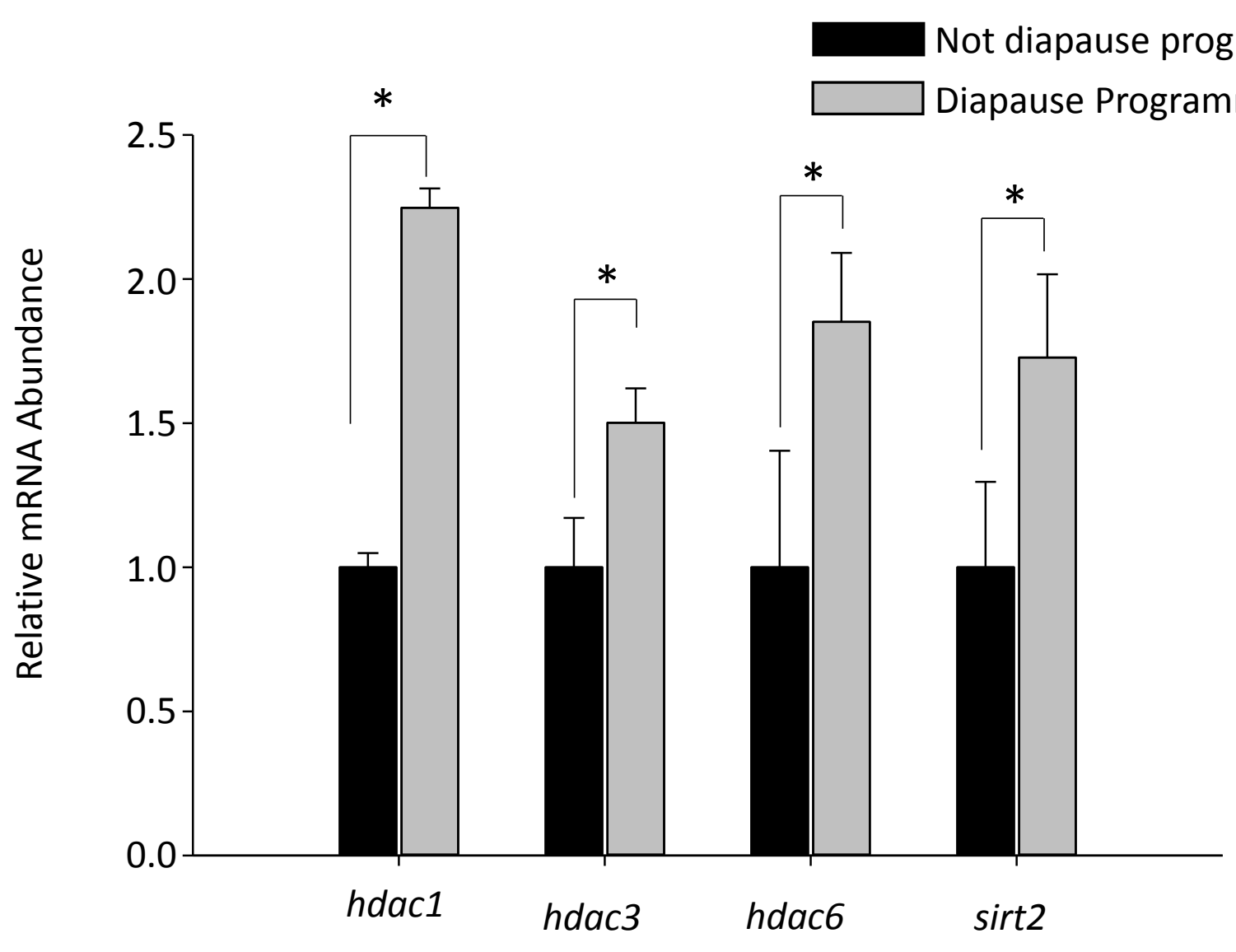

Figure 4 


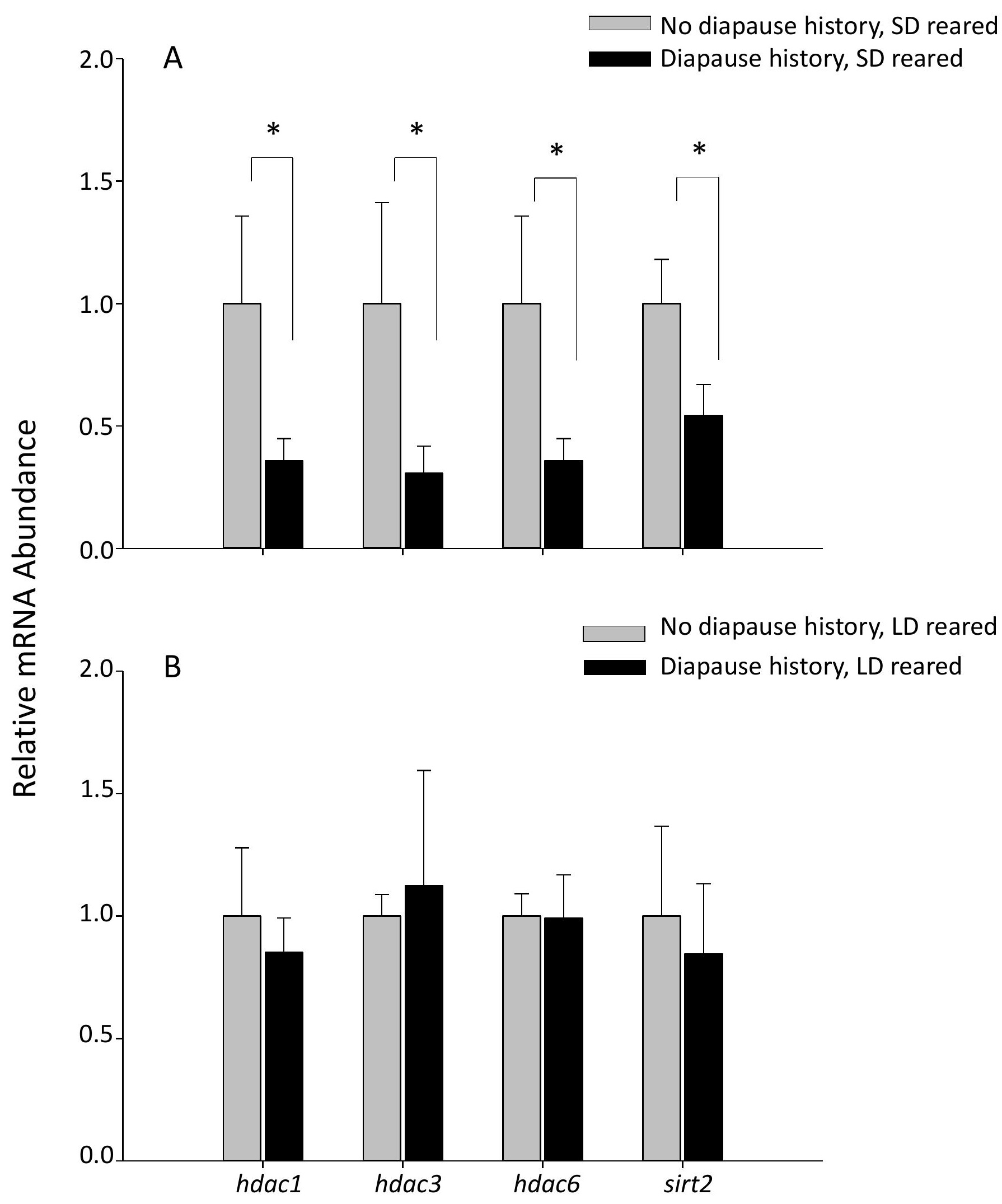

Figure 5 


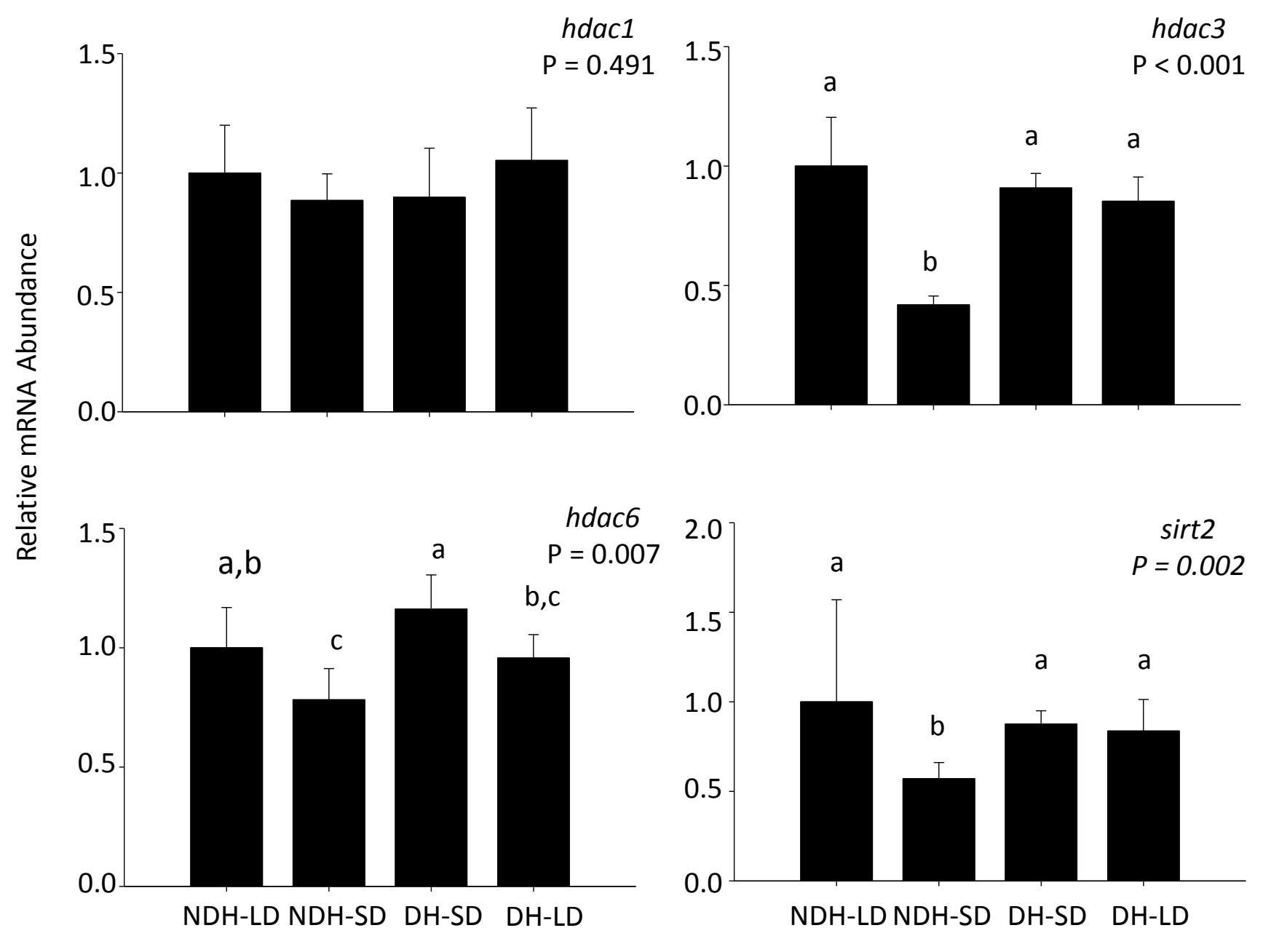

Figure 6 


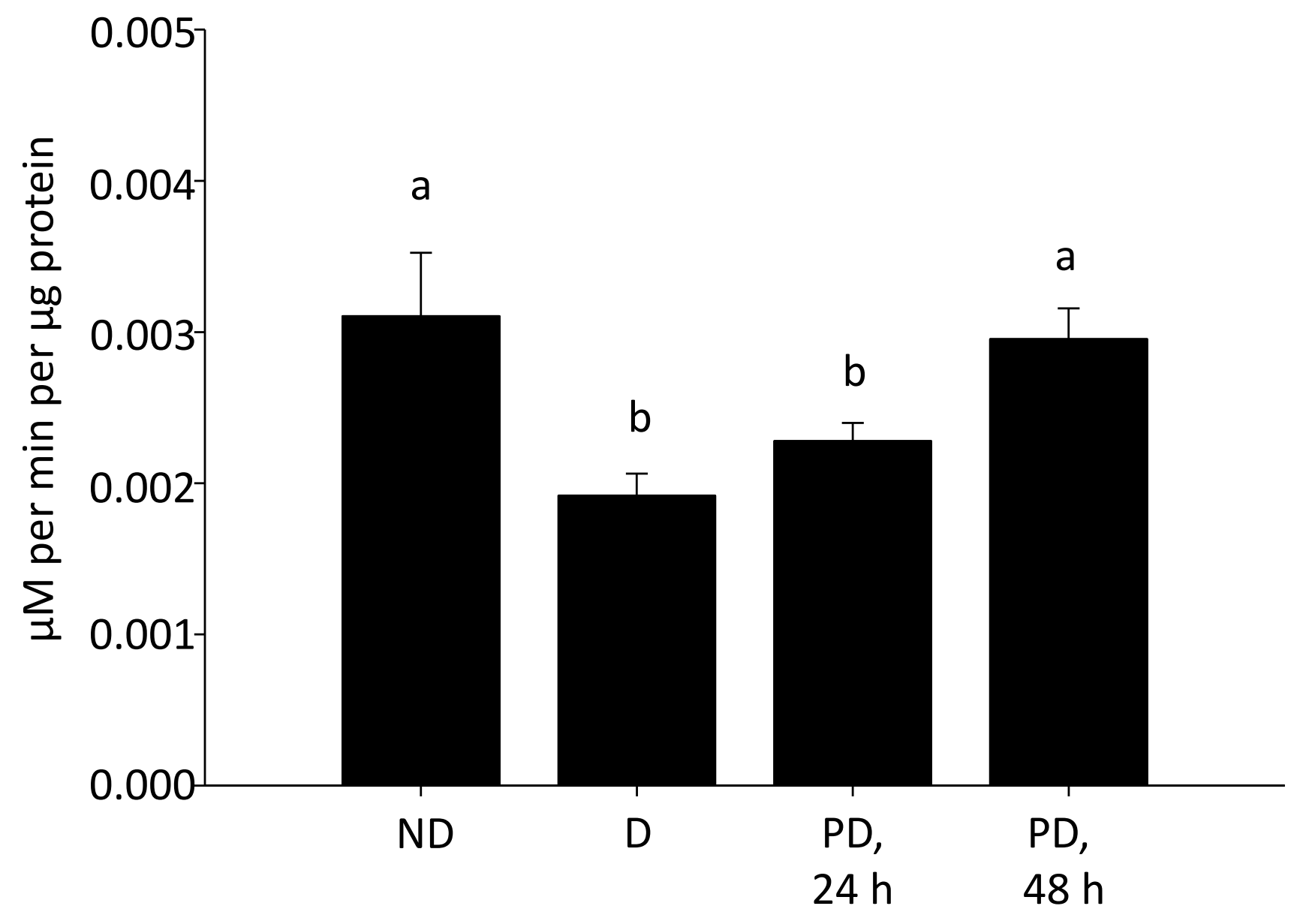

Figure 7 


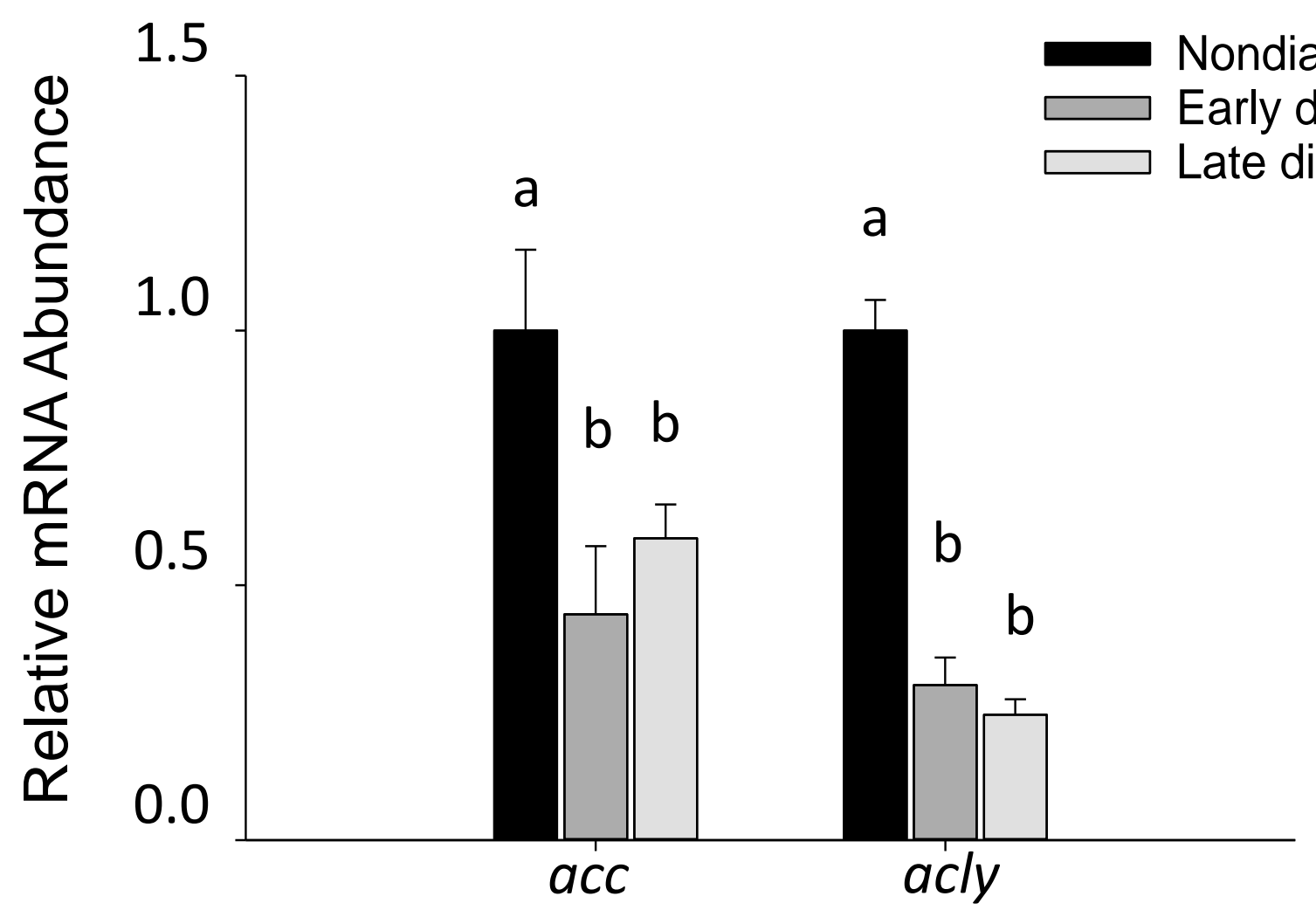

Figure 8 


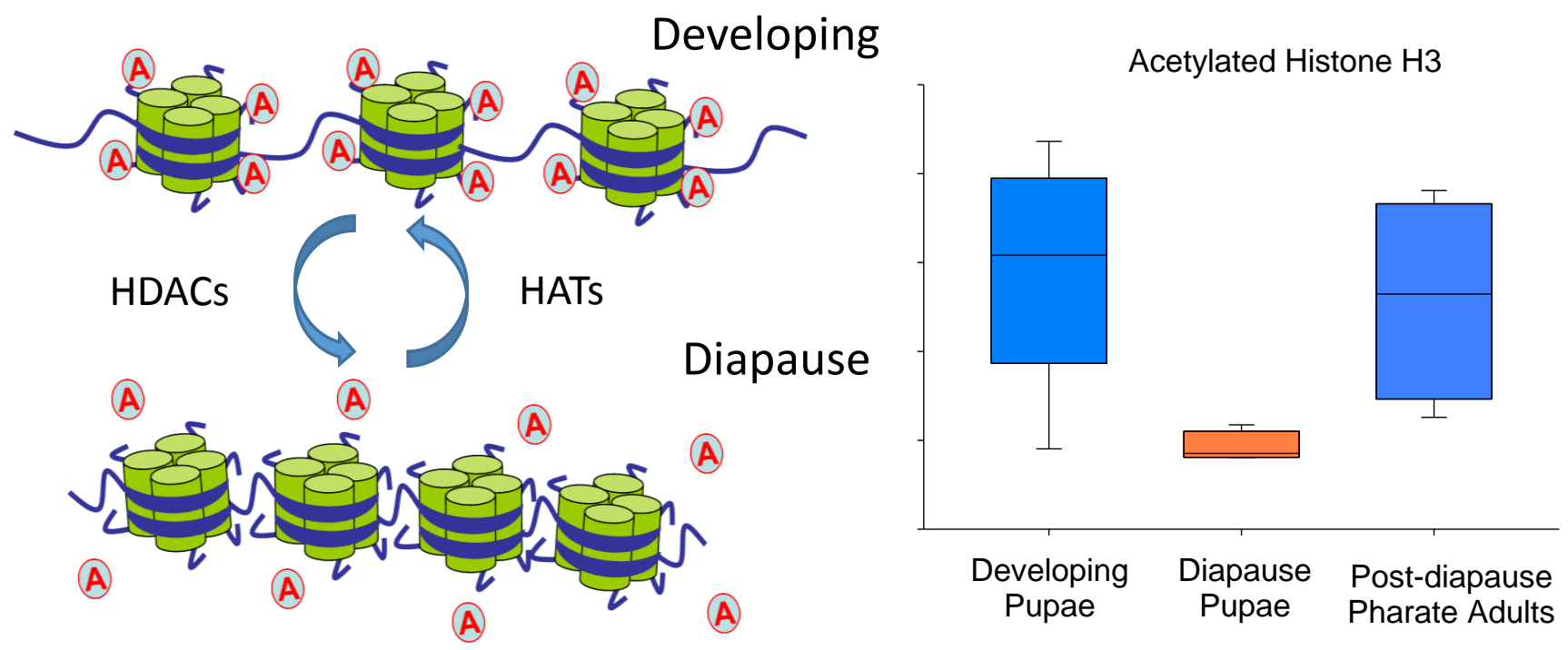

